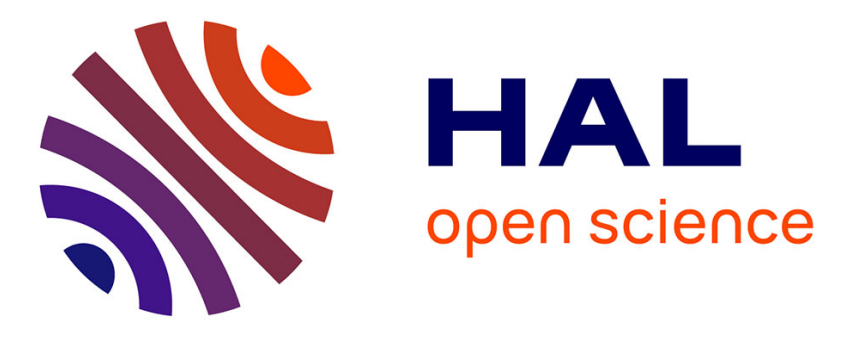

\title{
Compensation of resonant atomic dispersion using a pulse shaper
}

Jean-Christophe Delagnes, Antoine Monmayrant, Peter Zahariev, Arnaud Arbouet, Béatrice Chatel, Bertrand Girard, Mohamed A. Bouchène

\section{- To cite this version:}

Jean-Christophe Delagnes, Antoine Monmayrant, Peter Zahariev, Arnaud Arbouet, Béatrice Chatel, et al.. Compensation of resonant atomic dispersion using a pulse shaper. Applied Physics B - Laser and Optics, 2007, 86, pp.573-578. 10.1007/s00340-006-2549-7 . hal-00127178

\section{HAL Id: hal-00127178 \\ https://hal.science/hal-00127178}

Submitted on 29 Jan 2007

HAL is a multi-disciplinary open access archive for the deposit and dissemination of scientific research documents, whether they are published or not. The documents may come from teaching and research institutions in France or abroad, or from public or private research centers.
L'archive ouverte pluridisciplinaire HAL, est destinée au dépôt et à la diffusion de documents scientifiques de niveau recherche, publiés ou non, émanant des établissements d'enseignement et de recherche français ou étrangers, des laboratoires publics ou privés. 


\title{
Compensation of resonant atomic dispersion using a pulse shaper
}

\author{
J.C. Delagnes, A. Monmayrant, P. Zahariev, A. Arbouet, B. \\ Chatel, B. Girard, and M.A. Bouchene* \\ Laboratoire Collisions, Agrégats, Réactivité, (UMR 5589, CNRS - Université Paul \\ Sabatier Toulouse 3), IRSAMC, Toulouse, France
}

Fax: +33-561558317, E-mail: aziz@irsamc.ups-tlse.fr

The date of receipt and acceptance will be inserted by the editor

\begin{abstract}
An ultrashort pulse propagating in a resonant dense atomic medium experiences an important distortion due to a strong modification of its spectral phase. This distortion cannot be corrected using the usual simple dispersive devices (a pair of prisms, gratings, etc). We present here an experimental demonstration of the compensation of this effect using a dual 640-pixel high resolution pulse-shaper device. A cross-correlation intensity measurement combined with the XFROG (Cross-correlated Frequency Resolved Optical Gating) spectral phase measurement of the compensated pulse are performed; efficient correction of the resonant dispersive phase is shown. A spectacular temporal compression of the propagating pulse is then obtained.
\end{abstract}

PACS : 92.60.Ta; 42.50.Md; 42.65.Re; 42.79.-e

\section{Introduction}

Ultrashort optical pulses generally experience strong distortion when they propagate through dispersive media. In the weak field regime, these dispersion effects are entirely contained in the frequency dependence of the spectral phase $\phi(\omega)$ characterizing the transmittance of the medium. For laser frequencies far from any resonance, the medium is transparent and $\phi(\omega)$ is a slowly varying function which can be approximated by its polynomial expansion. The first and second order derivatives are the group delay and group velocity dispersion (GVD) respectively. The GVD induces a linear variation of the instantaneous frequency (chirp) and a temporal broadening of the pulse [1]. Compensation of this effect can be achieved with a pair of 
diffraction gratings or prisms with opposite GVDs $[2,3]$. On the other hand, the laser pulse is severely distorted when propagating through a medium with absorption resonances lying within the spectral bandwidth [4]. For instance, this situation occurs when a femtosecond pulse propagates in the atmosphere. The dispersion induced by the water vapor is then mainly responsible for the deformation of the pulse [5]. Since low order polynomial expansion of the spectral phase is no longer valid, the above-mentioned compressing devices are useless. Propagation of a chirped pulse through a resonant medium results in strong modulations due to interferences between the incident and the radiated field [6]. Distortion-free propagation is obtained for an incident field without any frequency components in the spectral region where dispersion occurs. For instance, this is the case of a train of ultrashort pulses where two adjacent pulses are locked with a relative phase of $\pi$ at the resonance frequency. As a result, the pulse-train propagates with very low distortion $[7,8]$. For different complex sequences, or a single pulse, compensation of resonant dispersion can obviously be achieved only with optical devices that can produce rapid variations of the spectral phase, such as pulse shapers [9]. They were successfully implemented in various fields such as coherent control $[10,11]$, quantum state and ultrashort electric field reconstruction [12-14] and optical communications [15].

In this paper, we show how the dispersion introduced by a resonant atomic medium on an ultrashort pulse can be compensated using such pulseshaper devices. The feasibility is experimentally demonstrated for considerably high optical depths $\left(\alpha_{0} \ell \sim 21000, \alpha_{0}\right.$ is the absorption coefficient at resonance and $\ell$ the length of the medium). Finally, we discuss the limitations of such a method.

\section{Basics of Ultrashort Pulse Propagation}

The temporal distortion of a Fourier-transform ultra-short pulse is constrained in the weak field regime by two propagation laws [16]. First, when the pulse is short, its spectral width is larger than the absorption line and so most of the initial energy is transmitted. Dispersion effects are prevailing and the quantity,

$$
W(z)=\int_{-\infty}^{+\infty} \varepsilon^{2}(t, z) \mathrm{d} t
$$

proportional to the pulse energy, is then constant $(\varepsilon(t, z)$ being the pulse envelope). The second propagation law is the McCall-Hahn area theorem which states that the pulse area decreases exponentially with the propagation distance $z$ as:

$$
\int_{-\infty}^{+\infty} \varepsilon(t, z) \mathrm{d} t=\mathrm{e}^{-\alpha_{0} z} \int_{-\infty}^{+\infty} \varepsilon(t, 0) \mathrm{d} t
$$


The pulse envelope thus develops an oscillatory temporal structure to satisfy these two conditions. The transmitted field after a length $\ell$ can be expressed as

$$
\varepsilon(t, \ell)=\frac{1}{\sqrt{2 \pi}} \int_{-\infty}^{+\infty} \tilde{\varepsilon}(\omega, 0) T(\omega, \ell) \mathrm{e}^{-i \omega t} \mathrm{~d} \omega
$$

where $\tilde{\varepsilon}(\omega, 0)$ is the Fourier transform of the incident pulse

$$
\tilde{\varepsilon}(\omega, 0)=\frac{1}{\sqrt{2 \pi}} \int_{-\infty}^{+\infty} \varepsilon(t, 0) \mathrm{e}^{i \omega t} \mathrm{~d} t
$$

and $T(\omega, \ell)=\mathrm{e}^{i \phi(\omega, \ell)}$ is the spectral field transmission of the atomic medium with

$$
\phi(\omega, \ell)=-\frac{\omega \ell}{c}(n(\omega)-1) \simeq-\frac{\alpha_{0} \ell \Delta_{d}}{\omega-\omega_{0}}
$$

( $n$ is the refraction index and $\Delta_{d}$ the Doppler width). Indeed, each frequency component $\omega$ experiences a phase shift which is significant $(\phi \geq 1)$ over a spectral range $\left[\omega_{0}-\alpha_{0} \ell \Delta_{d}, \omega_{0}+\alpha_{0} \ell \Delta_{d}\right]$. The real and imaginary parts of the spectrum exhibit strong oscillations for frequencies close to the atomic resonance (Fig. 1-a). In a simplified view of the process, the rapid oscillations of the phase give in the temporal domain small contributions that spread over a large temporal interval. The two side lobes separated by the spectral quantity $2 \alpha_{0} \ell \Delta_{d}$ lead to the main contributions, which result from beatings between these two frequency bands. Direct compensation of these dispersion effects is achieved with a pulse shaper introducing a spectral phase $\phi_{s}(\omega)$ opposite to the one introduced by the medium (see Fig. 1-b).

\section{Experimental Method}

The experimental demonstration of this effect is performed in atomic rubidium, on the $5 s^{2} S_{1 / 2} \rightarrow 5 p^{2} P_{1 / 2}$ transition at $\lambda_{0}=794.76 \mathrm{~nm}$. A home-built Ti:Sapphire femtosecond oscillator provides optical pulses with about 150 fs duration (Full Width Half Maximum (FWHM) of the intensity) and a spectrum bandwidth (FWHM) of $\Delta_{L}=23 \mathrm{rad} . \mathrm{THz}(7.7 \mathrm{~nm})$. A small fraction of the beam is used as a reference and the second part is successively sent into the pulse shaper along with a $12 \mathrm{~cm}$ long heat pipe containing an atomic vapor of rubidium. The pulse shaper consists of a zero dispersion line (4-f set-up) composed of one pair each of reflective gratings and cylindrical mirrors $[17,18]$. Its active elements are constituted by a combination of two 640-pixels liquid-crystal spatial-light-modulators (LC-SLM Jenoptik) located in the common focal plane of both mirrors of the 4-f line. Each pixel is $100 \mu \mathrm{m}$ wide. The LC-SLM has a high spectral resolution in both phase and amplitude. The spatial resolution of the pulse spectrum is $\delta=0.18$ rad.THz/pixel $(0.06 \mathrm{~nm} /$ pixel $)$ corresponds to a sufficiently wide temporal 
range (35 ps at $795 \mathrm{~nm}$ ). The temperature of the rubidium heat pipe is fixed at $160{ }^{\circ} \mathrm{C}$ for which the corresponding vapor pressure of rubidium is about $8.10^{-3}$ mbar. Argon is also used as a buffer gas at a pressure of 20 mbar.

The transmitted pulse is measured via intensity cross-correlation with the reference beam. Experimental results showing the temporal behavior of the pulse are represented in Fig. 2. In all cases, the dotted line curve represents the intensity profile of the laser pulse at the entrance of the medium and at the exit of the pulse shaper when the latter is not active. It represents the intensity profile to recover after pre-compensation by the pulse shaper and propagation in the heat pipe. The intensity cross-correlation is 250 fs FWHM and does not coincide with the FT duration corresponding to the spectral breadth. Results after propagation through the heat pipe are given in Fig. 2. In Fig. 2-a, the pulse shaper is not active and thus no phase compensation is introduced. The transmitted pulse exhibits oscillations due to the atomic dispersion that distorts the pulse profile. The experimental curve is fitted by a theoretical curve with the adjusted parameter $\left.\alpha_{0} \ell \Delta_{d}\right|_{\text {fit }}=13.6 \mathrm{rad} . \mathrm{THz}(4.55 \mathrm{~nm})$ obtained by a least squares fit method, which is in agreement with the calculated heat pipe characteristics (giving $\left.\alpha_{0} \ell \Delta_{d}\right|_{\text {calc. }}=13.4 \mathrm{rad} . \mathrm{THz}$ ). Fig. 2 -b represents the temporal profile of the laser pulse after propagation in the pulse-shaper device only. The phase shift requested from the pulse shaper is $\frac{\xi}{\omega-\omega_{0}^{\prime}}$ with $\xi=+12$ rad.THz and $\omega_{0}^{\prime} \simeq \omega_{0}$ within the spectral accuracy of the spectrometer ( $\sim 0.3 \mathrm{rad} . \mathrm{THz}$ or $0.1 \mathrm{~nm}$ ). In comparison with Fig. 2-a, the phase-inversion produces a time-reversed intensity profile. The experimental curve is fitted by a theoretical curve taking into account the actual phase and amplitude transmission of the pulse shaper and thus differing from the simple phase dependence requested. When the optical pulse propagates through both the heat pipe and the pulse-shaper, the dispersion effects cancel each other out and the distortion of the transmitted pulse is significantly reduced (Fig. 2-c, solid line). Note that the parameter $\xi$ above was chosen experimentally to achieve the best compensation effect. It does not coincide rigorously with the value $\left.\alpha_{0} \ell \Delta_{d}\right|_{\text {fit }}=13.6 \mathrm{rad} . \mathrm{THz}$ fitted from the best heat pipe data alone. The dashed line in Fig. 2-c represents the theoretical fit of the transmitted pulse taking into account both effects of the heat pipe and the pulse shaper. This will be discussed below in more detail.

\section{XFROG measurement - Phase reconstruction}

The compensation considerably suppresses the long ringing tail present after the pulses propagate through the atomic medium. Most of the dispersive spectral phase is obviously compensated in this experiment since the retrieved pulse is close to the initial pulse. Nevertheless, we have performed XFROG (Cross-correlated Frequency Resolved Optical Gating) measurements in order to quantify this conclusion. The signal emitted from the 
$\mathrm{BBO}$ crystal in the phase matching direction is then analyzed with a fibre coupled spectrometer. The fibre is injected with a $\mathrm{f}=5 \mathrm{~cm}$ focal length lens.

Fig. 3 depicts the raw XFROG data traces. We represent the XFROG signals of the output of (a) the pulse shaper programmed with the appropriate spectral phase for compensation, (b) the vapor cell alone, and (c) the combination of the two leading to compensation. Note the time inversion of the scale between Fig. 3-a and 3-b and c. In all cases (Fig. 3-a to Fig. 3-c), we have used a linear grey scale from 0 to 4500 with 512 levels. The three contour plots correspond respectively to 80,260 , and 1350 of the signal intensity. In this representation, we can see the correspondence between the lobes of each XFROG trace associated with the pulse shaper (Fig. 3-a) and the atomic vapor (Fig. 3-b). Despite the center positions as each lobe shifts progressively in time, there is a clear suppression of these lobes on a long time scale in Fig. 3-c. However a pedestal remains as observed in Fig. 2-c, 3 -c and 5-c.

This pedestal arises from the finite resolution of the pulse shaper which cannot compensate the divergence of the dispersion at resonance. Moreover the limited bandpass mostly affects the amplitude of transmitted spectral components even when a "phase-only" filter is applied. Indeed, large phase steps (e.g. " $\pi$-jump") between consecutive pixels lead to spectral holes in the transmitted amplitude [19] (see below). Before examining the spectral intensity of the transmitted pulse, let us consider the spectral phase extracted from the XFROG signal. The PCGPA (principal component generalized projections algorithm) [20] method is used to retrieve the complete set of amplitude and phase $\left(A_{s}(t), \phi_{s}(t)\right)$, and $\left(A_{g}(t), \phi_{g}(t)\right)$, where subscripts $\mathrm{s}$ and g denote for the "signal" and "gate" respectively. We resample the data into a square $2^{N} \times 2^{N}$ array (here $N=128$ ). The corresponding temporal and spectral resolutions are then $7.8 \mathrm{fs}$, and $1.1 \mathrm{~nm}$ respectively.

The spectral phases $\phi_{s}(\omega)$ and $\phi_{g}(\omega)$ are shown in Fig. 4. They are obtained by applying a FFT on the retrieved signal and gate. The two phases are represented over a pulsation interval of $\pm 21 \mathrm{rad}$. $\mathrm{THz}$ corresponding to twice the FWHM of spectral amplitude. Because the linear part does not affect the pulse shape it has been subtracted from the spectral phases $\phi_{s}(\omega)$ and $\phi_{g}(\omega)$.

On the interval presented here, $\phi_{g}(\omega)$ carries a phase fluctuation of 600 mrad (peak to peak). This important amount of fluctuation on the gate spectral phase is due to the presence of a noise on the associated temporal amplitude (not shown here) retrieved by the PCGPA. Indeed, the iterative method and convergence criteria of the reconstruction algorithm can distribute arbitrarily the noise between signal and gate [21]. In our reconstruction, the noise has a bigger contribution on the gate rather than on the signal. As for $\phi_{s}(\omega)$, the phase variation is $60 \mathrm{mrad}$, which is over the range where the spectrum amplitude is significant. This value indicates that the phase is sufficiently flat and compensated - within the resolution of $1.1 \mathrm{~nm}$ - and it explains the excellent results of the compensation in the temporal domain, especially the width of the main peak. 


\section{Limitation and alternative}

The discrepancy existing between actual and ideal compensations finds its origin in both the undersampling of the rapidly varying phase near resonance and the resulting modification of the amplitude of the transmitted spectral components. The former is due to the finite resolution of the pulse shaper (0.06 nm per pixel) which is unable to reproduce mask functions with characteristic scales of variation comparable or smaller than the pixel size. The latter is due to the finite spot size of each monochromatic component as a result of diffraction [22]. In general, pulse shapers are designed so that the spot size of each monochromatic component in the SLM plane is of the same order as the pixel size [23]. In the case of a slowly varying function, these two effects combine and achieve a smoothly varying function. In the opposite case, too rapid of a variation results in holes in the transmitted amplitude $[19,23]$. Because of the finite resolution (including diffraction limits in the Fourier plane), whatever the resolution of the device may be, there will always be a small spectral region where the phase dispersion cannot be reproduced correctly. Indeed, the oscillations are narrower and narrower when approaching the resonance frequency (Eq.4), and the pulse-shaper also significantly modifies the intensity near phase jumps.

The joint effect of diffraction and pixellation is significant if the phase variation between adjacent pixels is $\Delta \phi_{n}=\phi_{n+1}-\phi_{n} \geq 1$. From Eq.4 $\Delta \phi_{n} \simeq \delta \alpha_{0} \ell \Delta_{d}\left(\omega-\omega_{0}\right)^{-2}$ and is therefore significant in a spectral domain $\Delta_{\text {pix }}=2 \sqrt{\left(\alpha_{0} \ell \Delta_{d}\right) \delta}$ centered around the atomic resonance $\omega_{0}$, within which the transmitted electric field is strongly distorted. Introducing the quantity $\Delta_{\text {comp }}=\min \left(2 \alpha_{0} \ell \Delta_{d}, \Delta_{L}\right)$ over which the compensation has to be done, one needs $\Delta_{\text {pix }} \ll \Delta_{\text {comp }}$ to reduce the influence of these limitations and to ensure that distortions remain small. In our case $\Delta_{\text {pix }}=3.1 \mathrm{rad}$. THz $(1 \mathrm{~nm})$, $\Delta_{\text {comp }}=23 \mathrm{rad} . \mathrm{THz}$, and then $\Delta_{\text {pix }} / \Delta_{\text {comp }}=0.13$ indicating that $13 \%$ of the laser bandwidth is affected.

These effects contribute to the discrepancy observed near the atomic resonance between the theoretical phase requested, and that restored by the pulse-shaper (Fig. 1-b), and the attenuation of amplitude as shown in the transmitted spectrum (Fig. 1-c). Fig.1-c displays the transmitted intensityspectrum with the pulse shaper off (dotted line) or activated (solid line). The pulse-shaper significantly modifies the intensity near the atomic resonance [24]. Fig. 1-b and 1-c also confirm that the spectral domain over which this effect occurs is of the same order as $\Delta_{p i x}$ over which the phase variation is important. These discrepancies explain why the best compensation is obtained for a value of $\xi$ that is slightly different from the value of the optical thickness calculated from the experimental parameters or deduced from the cross-correlation in the non compensated case (Fig. 2-a).

The strong attenuation resulting from this effect can be avoided by replacing the rapid phase variation with a constant value on an interval of the order of $\Delta_{p i x}$ (Fig. 5-a). In this case, the amplitude transmission is greatly improved near the atomic resonance (Fig. 5-b). The temporal behavior of 
the transmitted pulse is shown in Fig. 5-c. The initial pulse is well restored and similar quality of compression is obtained when comparing to Fig. 2-c. However, the two methods are not equivalent. First, at the exit of the atomic medium, the phase of the spectral components near the central wavelength is almost compensated in the first case but not at all in the second. Secondly, more energy from the concerned region is transmitted in the second case, since the transmission is not altered. Thus, in the time domain, more energy is expected to spread out in the wings of the transmitted pulse. This effect is observed in Fig. 5-c, where a small peak appears near $600 \mathrm{fs}$.

\section{Conclusion}

We have shown that compensation of the resonant dispersion introduced by an optically dense atomic medium can be efficient for optical depths as high as $\alpha_{0} \ell \simeq 21000$ using a 640-pixel pulse shaper (note that for these optical depths, the attenuation at the line center is $\left.\mathrm{e}^{-21000}\right)$. Due to its finite resolution, the pulse shaper cannot follow the rapid phase variation in the vicinity of the atomic resonance. This limits both the quality of temporal focusing and most of the maximum optical depth that can be compensated. We tested two procedures of compensation. In one case, the energy spreading out of the central peak is reduced but the spectrum is profoundly altered near the atomic resonance. In the other case, the alteration of the spectrum is significantly reduced but more energy is left in the wings of the central laser peak. Depending on the fixed goal, one can use one or the other method of compensation. The compensation method was demonstrated on a single atomic resonance. The temporal focusing (compression) of ultrashort pulses is of general interest for selective excitation of a particular medium slice. For instance, it is used in nonlinear microscopy (THG) [25]. The realization of the ideas developed in the present paper is, in principle, feasible in the situation of propagation in an atmosphere containing numerous absorption lines due to the presence of water vapor. In this case, the spectral response can be rather complicated and/or difficult to describe analytically; an optimization algorithm could thus be used to achieve the best compensation monitoring the cross-correlation of the scattered signal. This might be considered in the future and would be of high interest for remote sensing. Indeed, the introduction of non-linearity in the detection scheme makes the latter sensitive to the peak intensity of the pulse. The position along the propagation axis at which the peak intensity is maximum, can be manipulated by changing the compensation introduced by the pulse shaper. This would give access directly to the local density of gas or particles.

Finally, the extension of this method to a non-linear interaction regime raises new possible investigations, since propagation effects also affect the intensity spectrum and/or spatial profile (self-phase modulation, filamentation). One can also use a pulse shaper to tailor an ultrashort pulse to control the light shift of an atomic system [26,27]. Moreover, the use of a 
pulse shaper in a closed loop scheme [9,28] may, for example, determine the form of incident shaped-pulses for which either distortion effects are minimized during propagation or spectral modifications fit a desired target shape.

We acknowledge financial support from the European Union Marie Curie program (Contract No. HPRN-CT-1999-00129, COCOMO).

\section{References}

1. J.-C. Diels and J.-C. Rudolph, Ultrashort laser pulse phenomena (Academic Press, New York, 1996).

2. E. Treacy, "Optical pulse compression with diffraction gratings," I.E.E.E. J. Quantum Electron. QE-5, 454 (1969).

3. R. L. Fork, O. E. Martinez, and J. P. Gordon, "Negative dispersion using pairs of prisms," Opt. Lett. 9, 150 (1984).

4. J. E. Rothenberg, D. Grischkowsky, and A. C. Balant, "Observation of the formation of the 0- $\pi$ pulse," Phys. Rev. Lett. 53, 552 (1984).

5. Y. Yamaoka, L. Zeng, K. Minoshima, and H. Matsumoto, "Measurements and Numerical Analysis for Femtosecond Pulse Deformations After Propagation of Hundreds of Meters in Air with Water-Vapor Absorption Lines," Appl. Opt. 43, 5523 (2004).

6. J. E. Rothenberg and D. Grischkowsky, "Measurement of the phase of a frequency-swept ultrashort optical pulse," J. Opt. Soc. Am. B 2, 626 (1985).

7. M. A. Bouchene, "Phase Control of Dispersion Effects for an Ultrashort Pulsetrain Propagating in a Resonant Medium," Phys. Rev. A 66, 065801 (2002).

8. M. Jacquey, S. Bonhommeau, and M. A. Bouchene, "Experimental observation of phase dependent propagation of an ultrashort pulse-train propagating in a resonant medium," Opt. Lett. 28, 1272 (2003).

9. A. M. Weiner, "Femtosecond pulse shaping using spatial light modulators," Rev. Sci. Inst. 71, 1929 (2000).

10. A. Assion, T. Baumert, M. Bergt, T. Brixner, B. Kiefer, V. Seyfried, M. Strehle, and G. Gerber, "Control of Chemical Reactions by Feedbackoptimized Phase-shaped femtosecond laser pulses," Science 282, 919 (1998).

11. J. Degert, W. Wohlleben, B. Chatel, M. Motzkus, and B. Girard, "Realization of a Time-Domain Fresnel Lens with Coherent Control," Phys. Rev. Lett. 89, 203003 (2002).

12. A. Monmayrant, B. Chatel, and B. Girard, "Femtosecond spectral electric field reconstruction using coherent transients," Opt. Lett. 31, 410 (2006).

13. A. Monmayrant, B. Chatel, and B. Girard, "Quantum State Measurement Using Coherent Transients," Phys. Rev. Lett. 96, 103002 (2006).

14. A. Monmayrant, B. Chatel, and B. Girard, "Real time quantum state holography using coherent transients," Opt. Commun. 264, 256 (2006).

15. H. P. Sardesai, C. C. Chang, and A. M. Weiner, "A femtosecond code-division multiple-access communication system test bed," Journal of Lightwave Technology 16, 1953 (1998).

16. M. D. Crisp, "Propagation of small-area pulses of coherent light through a resonant medium," Phys. Rev. A 1, 1604 (1970).

17. G. Stobrawa, M. Hacker, T. Feurer, D. Zeidler, M. Motzkus, and F. Reichel, "A new high-resolution femtosecond pulse shaper," Appl. Phys. B 72, 627 (2001). 
18. A. Monmayrant and B. Chatel, "A new phase and amplitude high resolution pulse shaper," Rev. Sci. Inst. 75, 2668 (2004).

19. W. Wohlleben, J. Degert, A. Monmayrant, B. Chatel, M. Motzkus, and B. Girard, "Coherent transients as a highly sensitive probe for femtosecond pulse shaper," Appl. Phys. B 79, 435 (2004).

20. D. J. Kane, "Recent progress toward real-time measurement of ultrashort laser pulses," IEEE J. Quantum Electron. 35, 421 (1999).

21. R. Trebino, Frequency-Resolved Optical Gating: The Measurement of Ultrashort Laser Pulses (Kluwer Academic, 2000).

22. M. M. Wefers and K. A. Nelson, "Analysis of programmable ultrashort waveform generation using liquid-crystal spatial light-modulators," J. Opt. Soc. Am. B 12, 1343 (1995).

23. The spot size is chosen to achieve a compromise between large spots who reduce the resolution and smaller spot size that causes the pixellated structure of the mask to appear as defects such as temporal rebounds or pedestal. The smoothing due to diffraction regards the complex number associated with the electric field of each monochromatic component, while the undersampling concerns the value of the phase. These two effects do not compensate for large phase steps. As an example, a phase step of $\pi$ results in attenuated transmitted amplitude for the components in the vicinity of the gap between the two adjacent pixels.

24. The strong variations induced on the transmitted intensity are smoothed because of the finite resolution of the spectrometer $(\simeq 0.1 \mathrm{~nm})$.

25. D. Oron and Y. Silberberg, "Harmonic generation with temporally focused ultrashort pulses," J. Opt. Soc. Am. B 22, 2660 (2005).

26. J. C. Delagnes and M. A. Bouchene, "Effect of a transient light shift on the propagation of an ultrashort pulse in a resonant atomic medium," Phys. Rev. A 69, 063813 (2004).

27. J. C. Delagnes, F. A. Hashmi, and M. A. Bouchene, "Spectral and temporal modifications of a weak resonant ultrashort pulse propagating in a two-level system driven by a strong nonresonant field," Phys. Rev. A (2006).

28. G. Heck, J. Sloss, and R. J. Levis, "Adaptive control of the spatial position of white light filaments in an aqueous solution," Opt. Commun. 259, 216 (2006). 